schen Eiskappe gerundet. Da und dort entstanden weite U-Täler, die in scharfen Gefällsbrüchen unvermittelt zum steilwandigen Fjord abstürzen. Tiefe Mündungsschluchten wurden seit der Eiszeit nur von wenigen Bächen geschaffen. Meist schießt das Wasser von der Hochfläche in Kaskaden zum Fjord hinab oder zerflattert als Fos (Wasserfall) an der steilen Felswand. Ein sehr schönes Beispiel dafür sind die «Sieben Schwestern» (Syv Söstre), sieben nebeneinander über die nördliche Wand stürzende Bäche. Im Bild hat das Schiff diese Stelle vor der Biegung soeben backbord passiert.

Fjordlandschaften sind wohl sehr malerisch; aber dies darf uns nicht über die kargen Lebensbedingungen hinwegtäuschen. Die Hochfläche liegt in dieser Breitenlage bereits über der Dauersiedlungsgrenze und kann nur noch «alpwirtschaftlich» genutzt werden (Säterwirtschaft) ; in den Fjorden selber sind jedoch die Siedlungs- und Wirtschaftsflächen beschränkt. Nur Verflachungen in weniger steilen Hängen, eiszeitlich abgeschliffene Sporne an der Einmündung kleiner Fjordarme oder nacheiszeitliche Deltas und Terrassen stehen landwirtschaftlicher Nutzung offen. Sorgsam mit Roggen, Hafer, Gerste oder Kartoffeln bepflanzte Äckerchen und kleine Heuwiesen sind aus topographischen und klimatischen Gründen (Besonnung) oft weit zerstreut - mit ihnen auch die vielen Einzelhöfe (Gaard). Die Siedlungskerne (Herred) bestehen in vielen Fällen nur aus Kirche, zwei bis drei Gehöften und manchmal Schule oder Kaufladen. Daß im Bild keine solchen Siedlungen zu erkennen sind, hängt mit der außerordentlichen Steilheit in diesem Abschnitt zusammen. Bereits wenige Kilometer fjordauswärts sind sie wieder vorhanden, desgleichen am Fjordende. Verbindungswege fehlen vielfach zwischen den abgelegenen Höfen. Nur über das Wasser des Fjords wird der Kontakt zu Nachbar und Außenwelt aufrechterhalten. Die Erwerbsbasis ist im allgemeinen so knapp, daß ein Zusatzerwerb auf dem Meer gesucht werden muß. So ergänzt - vor allem im Süden - der Fischfang während der Wintermonate (Hering, Kabeljau) die sommerliche Landwirtschaft.

Für den Nord-Süd-Verkehr sind die Fjorde ein arges Hindernis. Große Autofähren verbinden die fahrbaren Straßenstücke vor den Fjordausgängen und wichtigere Durchgangsrouten. Kleinfähren dienen weiter fjordeinwärts dem Lokalverkehr. Im Landesinnern verlaufen $\mathrm{Paßstraßen} \mathrm{über} \mathrm{die} \mathrm{öden} \mathrm{Fjell-Hochflächen,} \mathrm{oft} \mathrm{in} \mathrm{gewagten} \mathrm{Kehren}$ zu den Fjordenden absteigend. Gerade diese Stellen sind aber heute für den Tourismus bekannte Anziehungspunkte. Besonders geschätzt ist der 1955 fertiggestellte, $26 \mathrm{~km}$ lange Oerne-Vegen (Adlersweg), eine Aussichtsstraße, die vom hintersten Geirangerfjord nach Ytredal am oben erwähnten Nordalsfjord führt. Dank der tief ins Gebirge hineinreichenden Fahrwasser lassen sich weit im Landesinnern begeisternde See-LandReisen durchführen, die sich im Sommer stets wachsender Beliebtheit erfreuen.

\title{
VOM NILDELTA
}

\author{
ERNST LEEMANN
}

Das Wort Herodots, daß Ägypten ein Geschenk des Nils sei, gilt heute wie jemals; denn ohne das Bewässerungswunder wäre das Niltal Wüste wie seine Nachbarschaft in Ost und West.

Der Nil hat sich im Laufe der Zeit, wie jeder andere Fluß, in die östliche Wüstentafel der Sahara eingeschnitten. Fünf Etappen sind durch ebensoviele Erosionsterrassen festgestellt. Auf der zweitobersten, also schon in den Anfängen der Talbildung, ist paläolithische Besiedlung durch Funde von Steinwerkzeugen sichergestellt. Die Besiedlung hat also auch vorhistorisch hier ein hohes Alter. 


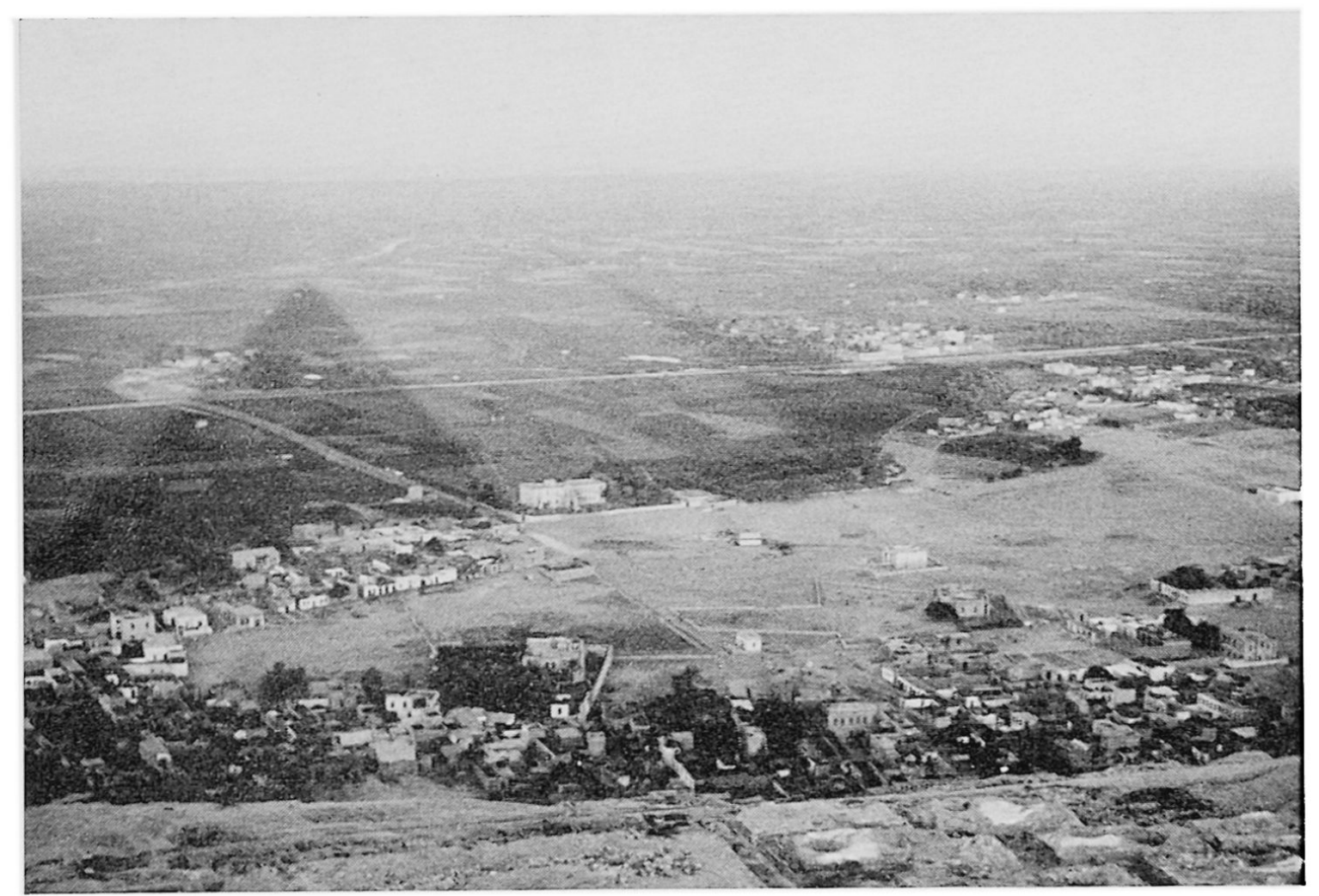

Abb. 1: Aussicht von der Cheopspyramide. Bildrand unten: Rand des erhöhten Wüstenplateaus, links: Schatten der Pyramide in der Nilebene, links oben: schmaler Streifen des Flusses.

In rund $1200 \mathrm{~km}$ langem Lauf durchfließt der Nil sein ägyptisches Tal, um bei Kairo, $175 \mathrm{~km}$ vom Meer entfernt, aus der Umklammerung der Randketten in die große Deltazone auszutreten. Einheitliche Landschaftsformen, wie klimatische und vegetative Erscheinungen begleiten den Fluß auf seiner ganzen Länge. Der Gegensatz zwischen der grünen Stromoase und der benachbarten Wüste tritt überall in aller Schärfe zutage. Nicht nur von der Spitze der Cheopspyramiden kann man die Grenze zwischen dem begrünten Talboden und den Wüstenplatten nahezu als scharfe Linie erkennen, sondern oft auch längs der Bahnlinie Luxor-Assuan, wo zur einen Seite die fruchtbare Talebene liegt, zur andern aber der Wüstensand sein Reich ausgebreitet hat.

Erdgeschichtlich ist nachgewiesen, daß der Ur-Nil im Tertiär die östliche libysche Wüste durchfloß. Entsprechende Ablagerungen sind an verschiedenen Orten festgestellt. Aber im Pliocän erfolgten einige Einbrüche in den umliegenden Gesteinstafeln, in denen sich die Talbildung nunmehr weiter entwickelte und das heutige System schuf.

Bei $30^{\circ} \mathrm{N}$, also direkt bei Kairo, sinken die beidseitigen Randhöhen zur Mündungsbucht, dem heutigen Delta, ab, und trichterförmig weiten sich die Ablagerungen des Nils, fruchtbares Land schaffend, nach allen Seiten aus. Der englische Historiker Toynbee schreibt in seinem Werk: «Der Gang der Weltgeschichte» über das Nildelta: «Zuerst hatten sich die Bewohner der Bewältigung einer furchtbaren Umwelt zu widmen: der Rodung, Entwässerung und der Kultivierung des Dschungelsumpfes, der ursprünglich das untere Niltal und das ganze Delta einnahm und den Menschen ausschloß».

Der Höhepunkt der wirtschaftlichen und politischen Triebkraft des Volkes wurde in der vierten Dynastie erreicht, in der die Urbarmachung der Sümpfe bereits so weit vorgeschritten war, daß die mächtigen Pyramidenbauten in Angriff genommen werden 


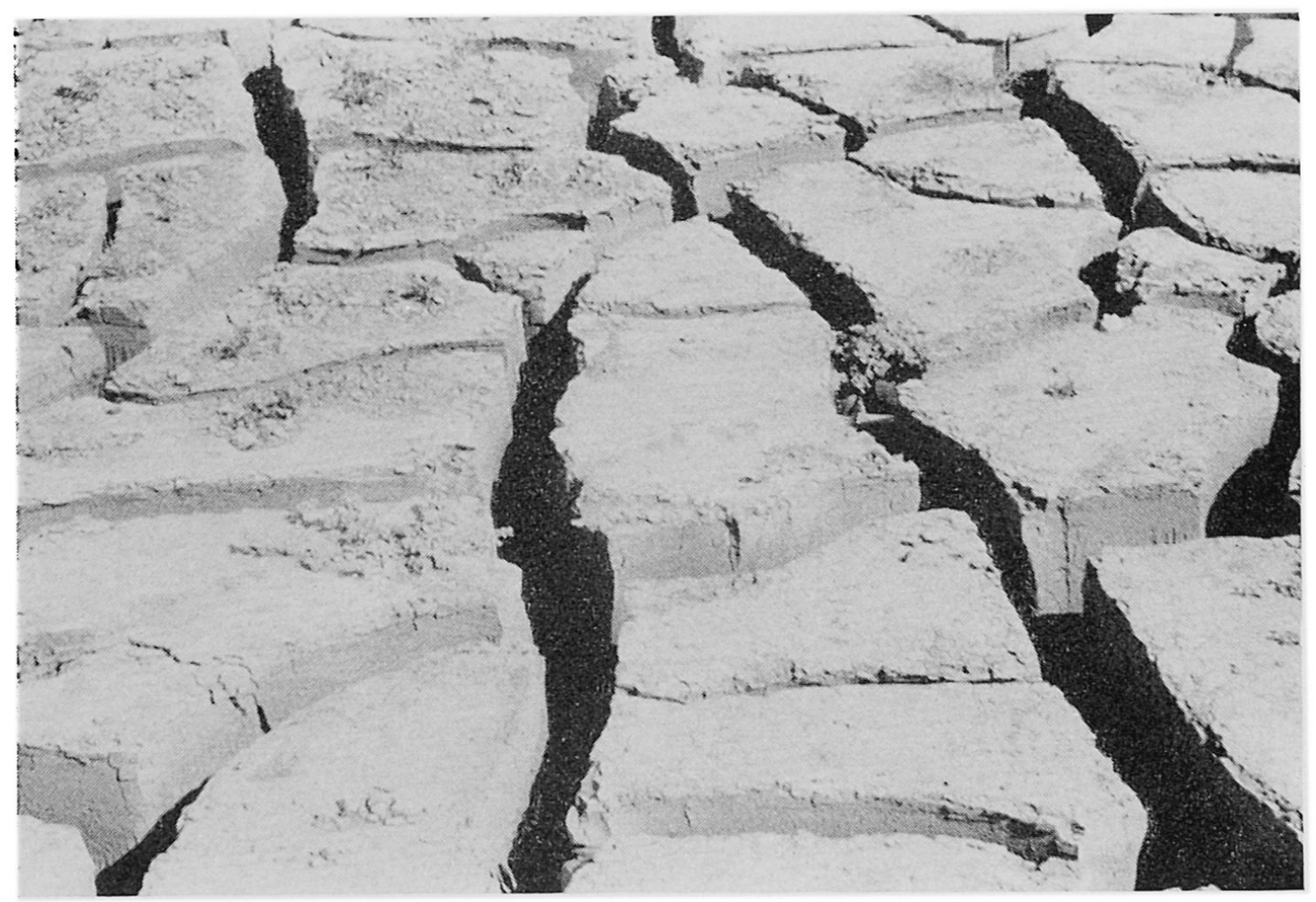

Abb. 2: Nilschlammboden. In trockenen Fluß- oder Kanalstrecken hat sich fruchtbarer Nilschlamm angesammelt, der bis $12 \mathrm{~m}$ Tiefe erreichen kann.

konnten. Das alte Reich, von dem hier die Rede ist, wird in die Zeit von 2900-2370 v. Ch. eingegliedert.»

Das Delta mißt rund $250 \mathrm{~km}$ von Wesi nach Ost und $175 \mathrm{~km}$ in Nord-Süd-Richtung. Auch heute noch schieben sich die Mündungsarme bei Rosette und Damiette jährlich um vier Meter meerwärts vor, trotzdem durch den Bau der Staudämme die Schlammführung zum Meer stark reduziert worden ist. Der Küste entlang zieht sich eine amphibische Landschaft hin. Vier größere und zahlreiche kleinere, fisch- und vogelreiche Strandseen, die durch schmale Landzungen vom Meer getrennt sind, lassen das Wachsen des Deltas erkennen, und umliegendes, brackisches Sumpfgelände bezeugt anschaulich das Landwerden auch in rezenten Zeiten.

Im Altertum erreichte das Nilwasser in sieben Armen das Meer. Entgegen der heutigen Situation, wo es eigentlich nur noch deren zwei sind und die ziemlich in der Mitte des Deltas meerwärts strömen, erreichten die damaligen Flußarme eher im Westen und Osten die Küsten. Noch können die kanopische, bolbitische, sebenitische, phatnitische, mendesmitische, tanitische und pelusische Mündung in alten Karten gut erkannt werden, und noch sind einige dieser Arme als Bewässerungskanäle in Funktion.

Wenn man sich mit dem Schiff vom Meere her der afrikanischen Küste nähert, bietet sich der Kontinent in wenig imponierender Art dar, denn hinter einem schmalen Sandstrand steigt eine wenig ausgeprägte, niedere Steilküste auf. Das Deltaland präsentiert sich als weite, einförmige Ebene, die erst in der Nähe ihre besondern Reize zu erkennen gibt. Da und dort unterbricht ein Haufen niederer Lehmhäuser, zu einem ärmlichen Fellachendorf geschart, von spärlichen Sykomoren- oder Palmgruppen umrahmt, die gleichförmige Ebene. Über der Grabmoschee des Dorfheiligen steigt ein weißes, schlankes, niederes Minarett in das sonnige Blau des Himmels auf. 


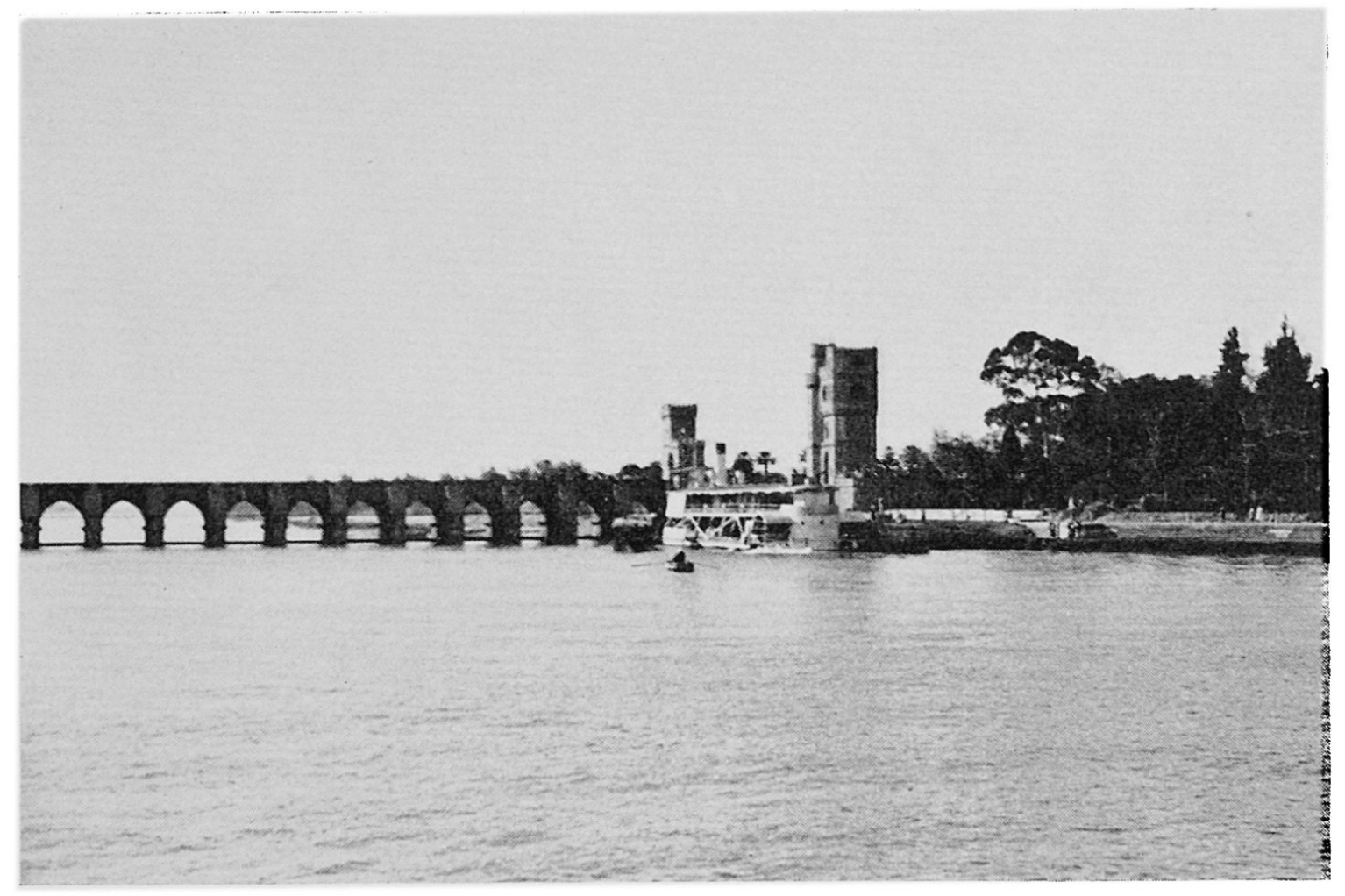

Abb. 3: Barrage du Nil. Der alte Damm regelt den Abfluß ins Delta.

Zahlreiche alte Flußläufe und zum 'Teil ausgetrocknete Kanäle durchmessen das Land. Sie bringen das begehrte Wasser in alle Zonen des $31000 \mathrm{qkm}$ großen Deltas und bieten bei genügender Wasserführung zugleich willkommene Transportmöglichkeiten. Von hochragenden spitzen Segeln getrieben, gleiten die breiten Lastkähne gemächlich dahin. Oft herrscht aber völlige Windstille. Dann stehen die Menschen auf den Dämmen in die Schleppseile, um die breitbauchigen Schiffe vorwärtszubringen. Schwerbeladene Kamele kommen auf den Dammwegen daher, von flinken Eselreitern rasch überholt. Kleine Herden von Rindern und Ziegen suchen auf dürftigen Weiden oder Stoppelfeldern kärgliche Nahrung. Esel sind rarer und Kamele gar spärlich. Im Schlamm halbtrockner Kanäle wälzen sich schwarze Büffel in wohligem Zustand. Wenn auch angesichts der großen Entfernungen ausgedehnte Landstriche verkehrsgeographisch ungünstig liegen und darum die 'Transportmöglichkeiten für die angebauten Produkte unzweckmäßig sind - denn auch größere Kanäle liegen bislang vom Februar bis zum August trocken -, ist doch die ganze Deltaebene reich bebaut. Man kann wohl sagen, daß jeder Quadratmeter Boden bestellt ist: grünende Saaten im Winter, gelbblühende Baumwollfelder im Sommer, weiß aufquellende Kapseln im Herbst, Orangen und Mandarinen im Winter, Bananen das ganze Jahr und reifende Datteln an eleganten Hochstämmen im November.

Das Wunder der Fruchtbarkeit muß heute teuer erkauft werden, denn lang ist es her, daß die herbstliche Nilflut aus eigenem Drang das Land mit ihrem Nährschlamm überflutete und überall, wo das Wasser hinzukommen vermochte, Frucht und Nahrung schuf. Heute sind die eigentlichen Überschwemmungsgebiete fast ganz verschwunden, und die frühern Ablagerungen des Nilschlammes, die bis $12 \mathrm{~m}$ Tiefe erreichen, werden kaum mehr zunehmen. Mit dem im Jahr 1821 erfolgten Großanbau der Baumwolle wurde Dauerbewässerung nötig, was nur durch den Bau von Staudämmen und neuer- 
liche Vertiefung alter Kanäle möglich wurde. Im Jahr 1835 wurde der «Barrage du Nil» unterhalb Kairo in Angriff genommen, aber tatsächlich erst 1890 vollendet. Die Regulierung betrifft die beiden Mündungsarme und die wichtigsten Kanäle.

Drei Hauptkanäle, die im wesentlichen alten Mündungsarmen folgen, speisen das Deltaland. Zwischen ihnen verteilen unzählige Rinnsale das Berieselungswasser, das mit den verschiedensten Hebewerken auf den Ackerboden gebracht wird. Besonders niedrig gelegene Flächen können bei höhern Wasserständen auch heute noch überschwemmt werden. Auf der abgelagerten Schwemmerde, die viel Kali, aber wenig Nitrate enthält, können bei entsprechender Düngung hohe Erträge erzielt werden.

Trotz den verschiedenen Dammbauten tritt in den Monaten März bis Mai Wassermangel auf, der erst behoben ist, wenn anfangs Juli der alte Wassermesser auf der Insel Roda bei Kairo das Steigen des Nils verkündet. Neben modernen Pumpanlagen wird mit dem alten Schâdûf, der knarrenden Sâkije, dem alten Tâbût und dem Tanbûr, also mit Holzfächerrädern und mit archimedischen Schrauben, das Wasser auf die Landoberfläche gehoben. So ist hier der Nil heute wie in alten Zeiten der Vater des Landes. Erst die Vollendung des neuen Assuanhochdammes wird auch dem Delta verbesserte Bewässerungsmöglichkeiten bringen.

Die Deltaböden stellen die wertvollsten Landesteile Ägyptens dar. Sie bilden die eigentliche Versorgerbasis für den Großteil der Bevölkerung. Heute aber sind sie so weit abgenützt, daß ohne Brache oder regenerierende Fruchtfolgen und Düngung keine hohen Erträge mehr erzielt werden können. Immerhin sind in bestimmten Anbauzyklen immer noch zwei Ernten möglich; z. B. Weizensaflor (eine Färbereidistel) oder Weizenklee. Da Kuhmist wegen der Holzarmut des Landes als Brennmaterial dient und der Taubenmist der Taubenhäuser nur für Gärten ausreicht, muß viel Kunstdünger importiert werden, zumal die neu errichteten Kunstdüngerwerke am Obernil den Landesbedarf nicht zu decken vermögen.

Vorwiegend drei jahreszeitliche Kulturen geben der Landwirtschaft das Gepräge:

1. Winterkultur. Anfangs Oktober erfolgt die Aussaat für Weizen, Bohnen und Klee; im Mai ist Erntezeit.

2. Sommerkultur. Reis und Baumwolle, Aussaat im Mai, Ernte im Oktober.

3. Herbstkultur. Zwischensaat und Ernte für Mais, der im Delta Hauptnahrung ist. Die Kultur benötigt 2 $\frac{1}{2}$ Monate.

Reis kann nur in der unmittelbaren Nachbarschaft zuverläßig dauernd wasserführender Kanäle gebaut werden; auch Zuckerrohr nimmt keinen wesentlichen Raum ein. Die Baumwolle, die schon im Altertum bekannt war, wird seit der Mitte des vorigen Jahrhunderts intensiv angebaut und ist heute hier eigentliche Hauptkultur. Sie bedeckt gut ein Drittel der Bodenfläche. Motorpflüge und Entkörnungsmaschinen sind längst eingeführt, denn die Baumwolle ist ein bedeutsamer Exportartikel. Dem ägyptischen Arbeitsministerium ist zu ihrer Förderung ein spezielles Baumwollforschungsinstitut angegliedert. Im Jahr 1962 betrug der Ausfuhrwert der Rohbaumwolle 122,7 Millionen ägyptische Pfund, derjenige der Baumwollgarne 26 Millionen Pfund.

Natürlich werden noch viele andere Produkte angebaut, so Hülsenfrüchte, Gemüse, Oelfrüchte, Gewürze, Färbstoffe (z. B. das altbekannte Henna). Gegen 30 Dattelarten gedeihen im Land, die meist in den Inlandkonsum gehen. Bevorzugt sind die Spätdatteln von Alexandria.

Zum Klima sind kaum viele Hinweise nötig, denn jedermann kennt den ewig blauen Himmel, die goldene Sonne, die trocken-reine Luft, wenn man nicht gerade auf staubigen Dorfstraßen unterwegs ist. $\mathrm{Da}$ es im Delta im Winter etwas trüber und feuchter ist, entspricht der Nähe des Mittelmeeres, dessen frische Brisen vorab im Sommer, wenn die Glut der Wüste über dem Lande lastet, recht willkommen sind.

Man könnte erwarten, daß auf diesem Deltaboden, der an den Berührungslinien dreier Kontinente liegt und tatsächlich seit den ältesten Zeiten der Menschheit Durch- 


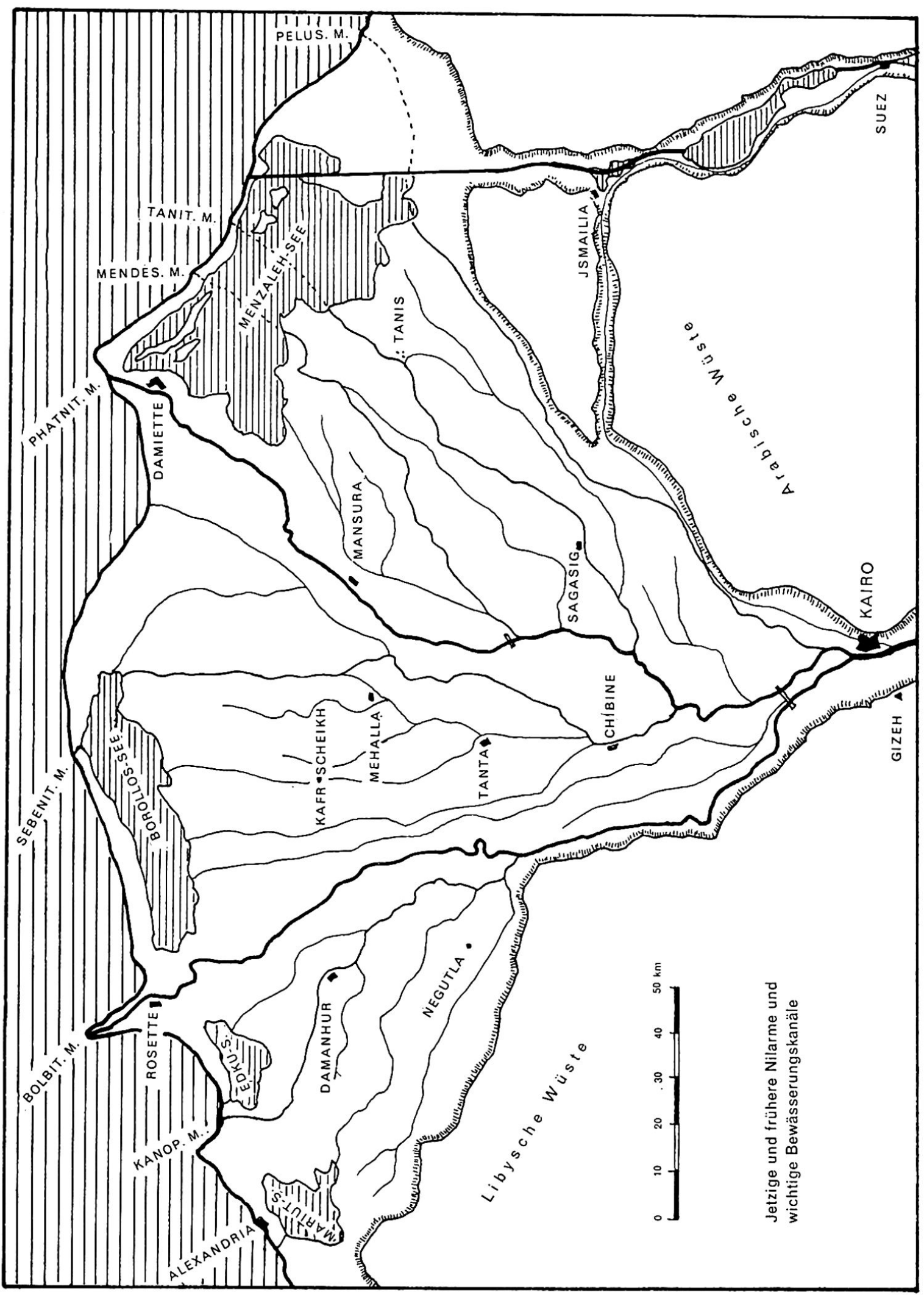

Abb. 4: Gewässerkarte des Deltas. In ihr sind ältere und heutige Nilarme und Bewässerungskanäle eingezeichnet; auch die sieben alten Nilmündungen, die heute noch teilweise als Kanäle funktionieren, sind angegeben. 
gangsland war - hier brachen asiatische Nomaden, Hebräer, Hyksos, Assyrer, Babylonier und Perser ein, über das Meer erschienen Griechen und Römer, von Süden drängten afrikanische Stämme, und von Osten nahm auch der Islam diesen Weg - ein unentwirrbares, rassisch verknäueltes Völkergemisch vorherrschen müßte. Aber nichts von alledem bis auf geringe Nuancen, die in kleinen Unterschieden des Haus- und Feldbaues zwischen dem offenen Osien und dem eher von diesen Völkerschüben abgewandten Westen zu erkennen sind. Auch mag hier der Menschenschlag stärker ans Beduinische anklingen, wenn auch der Fellache überall den Kern der Bevölkerung darstellt.

Diese Menschen, von bewundernswertem Fleiß und grenzenloser Einfachheit, sind Meister ihres Handwerkes. Nicht nur der guten Erde sind die hohen Erträge zuzuschreiben, wohl ebenso dem angestammten Können seiner Bewohner. Diese hellhäutigen, robust gebauten Bauern wohnen in armseligen Hütten, die sich oft in stadtähnlichen Gassen zu Häuserzeilen reihen. Die aus luftgetrockneten Schlammziegeln gebauten Wände werden von einem schüttern Dach aus Durrastroh überdeckt, das kaum Schutz vor Regen zu bieten sich bemüht, was vielleicht im ägyptischen Sonnenland einem Dach auch nicht zugemutet werden kann. Dem Ackerbauer ist die Sonne heilig, und aus innerer Beziehung folgt er ihrem Jahreslauf, der sein ganzes Leben und den Ablauf aller Dinge auf dem Boden seiner Ahnen lenkt und lohnt.

Dürftig wie sein Haus sind Kleidung und Hausrat. Ein paar Felle, dünne Matten, etliche Holzschüsseln, Kessel und Tonkrüge bilden sein Inventar. Entsprechend einfach, wenn auch kalorienmäßig genügend, ist seine Ernährung nach dem Motto: schnell, wenig, schlecht. Maisbrot, Saubohnen, eine warme Speise aus Zwiebeln, Leinund Sesamöl soll tägliches Abendbrot sein. Von Ziegen gewinnt man Milch, die in gesäuertem Zustand genossen wird, offenbar mehr aus Not als aus Genuß. Etwas Grünzeug, viel Gurken und Kürbisse ergänzen die Tageskarte. Auch auf den Bahnhöfen werden den Reisenden Gurken angepriesen, deren saftiges Fleisch den Durst stillt.

Früher war der Boden restlos in den Händen der Großgrundbesitzer, was durch die neuern Bodenreformen einigermaßen gemildert worden ist im Zuge staatlicher Maßnahmen, die auf dem Papier sehr weitgreifende Änderungen vorsehen. Vorerst ist noch nicht viel geschehen in dieser Richtung. Im Jahre 1912 hatten die Engländer das FünfFeddân-Gesetz eingeführt, das auch heute noch Gültigkeit hat. Es bestimmt, daß ein Besitz von fünf Feddân Boden (2,1 ha) nicht gepfändet werden kann, wodurch wenigstens dem gemeinen Wucher der Riegel einigermaßen gestoßen worden war.

Das Delta war zu allen Zeiten gut besiedelt. Viele aufblühende Dörfer und Städte sind Zeugen des landwirtschaftlichen Aufschwungs. Auch um die Hebung des kulturellen Volksniveaus ist man ehrlich bemüht durch Schaffung von Schulen aller Stufen.

Natürlich begegnet auch der Geograph der altägyptischen Kunst mit Interesse, doch ist hier kaum der Ort zu entsprechenden Erörterungen, um so weniger, als die Ausgrabungen alter Kultstätten im Delta zahlenmäßig noch geringfügig sind. Trotzdem deuten zahlreiche kleinere Hügel, Tell genannt, auf alte Bauten hin. Auch durch die Überlieferung sind alte Siedlungen und Kulturstätten an vielen Orten gesichert.

\section{ON THE NILE DELTA}

At Cairo the Nile leaves the hills that have so far bordered it, and for the remaining $175 \mathrm{~km}$ there is the big delta, extending over $31000 \mathrm{qkm}$. While today the Nile reaches the sea in two branches of an estuary, there were seven of them in antiquity. Some are still in use as irrigationchannels, some as water-ways for small cargo-boats. It is above all cotton that needs adequate moistness, but there are other cultivations that depend on irrigation in the dry season, as for example corn, beans, clover, rice, maize and sugar-cane.

The delta-farmers are hard-working and efficient people, living in simple huts. The new landreforms try to improve their lot by gradually abolishing the old feudal system and to tie them more effectively to the country by giving them their own piece of land. 